\title{
FUNCTION CLASSES FOR SUCCESSFUL DE-SINC APPROXIMATIONS
}

\author{
KEN'ICHIRO TANAKA, MASAAKI SUGIHARA, AND KAZUO MUROTA
}

\begin{abstract}
The DE-Sinc formulas, resulting from a combination of the Sinc approximation formula with the double exponential (DE) transformation, provide a highly efficient method for function approximation. In many cases they are more efficient than the SE-Sinc formulas, which are the Sinc approximation formulas combined with the single exponential (SE) transformations. Function classes suited to the SE-Sinc formulas have already been investigated in the literature through rigorous mathematical analysis, whereas this is not the case with the DE-Sinc formulas. This paper identifies function classes suited to the DE-Sinc formulas in a way compatible with the existing theoretical results for the SE-Sinc formulas. Furthermore, we identify alternative function classes for the DE-Sinc formulas, as well as for the SE-Sinc formulas, which are more useful in applications in the sense that the conditions imposed on the functions are easier to verify.
\end{abstract}

\section{INTRODUCTION}

The Sinc approximation formula, expressed as

$$
f(x) \approx \sum_{k=-N}^{N} f(k h) S(k, h)(x)
$$

is an interpolation formula to approximate a function $f$ on the real line $\mathbf{R}$ based on sampled values $\{f(k h) \mid-N \leq k \leq N\}$ at a finite number of equally-spaced points on $\mathbf{R}$, where $N \in \mathbf{N}$ and $h>0$. Here $S(k, h)$ denotes the Sinc function defined as

$$
S(k, h)(x):=\frac{\sin [\pi(x / h-k)]}{\pi(x / h-k)} .
$$

The formula (1.1) is known to achieve very high accuracy if $f$ is a well-behaved function decaying sufficiently rapidly as $|x|$ tends to infinity. Numerical methods based on this Sinc approximation, initiated by McNamee, Stenger and Whitney 2], have been developed and applied to various scientific computations in the last three decades. They are now accepted under the name of Sinc numerical methods [6, 7, 11.

Received by the editor February 8, 2007, and in revised form, June 19, 2008.

2000 Mathematics Subject Classification. Primary 65D05; Secondary 41A25, 41A30.

Key words and phrases. Sinc approximation, double exponential transformation.

This work was supported by the 21st Century COE Program on Information Science and Technology Strategic Core and a Grant-in-Aid of the Ministry of Education, Culture, Sports, Science and Technology of Japan. The first author was supported by the Research Fellowship of the Japan Society for the Promotion of Science for Young Scientists. Technical details omitted in this paper can be found in [14]. 
The formula (1.1) can be adapted to approximations on general intervals with the aid of appropriate variable transformations $x=\psi(t)$. When $f$ is approximated on an interval $I \subseteq \mathbf{R}$, the formula is modified to

$$
f(x) \approx \sum_{k=-N}^{N} f(\psi(k h)) S(k, h)\left(\psi^{-1}(x)\right)
$$

with a transformation function $\psi: \mathbf{R} \rightarrow I$. This approach works if $\psi$ is chosen appropriately so that the transformed function $f(\psi(\cdot))$ satisfies certain conditions, say, about the decay rate.

As for the transformation function $\psi(t)$ we can employ an appropriate double exponential (DE) transformation such as

$$
\begin{aligned}
& \psi_{\mathrm{DE} 1}:(-\infty, \infty) \rightarrow(-1,1), \quad \psi_{\mathrm{DE} 1}(t):=\tanh ((\pi / 2) \sinh t), \\
& \psi_{\mathrm{DE} 2}:(-\infty, \infty) \rightarrow(-\infty, \infty), \quad \psi_{\mathrm{DE} 2}(t):=\sinh ((\pi / 2) \sinh t), \\
& \psi_{\mathrm{DE} 3}:(-\infty, \infty) \rightarrow(0, \infty), \quad \psi_{\mathrm{DE} 3}(t):=\exp ((\pi / 2) \sinh t), \\
& \psi_{\mathrm{DE} 4}:(-\infty, \infty) \rightarrow(0, \infty), \quad \psi_{\mathrm{DE} 4}(t):=\exp (t-\exp (-t)), \\
& \psi_{\mathrm{DE} 5}:(-\infty, \infty) \rightarrow(0, \infty), \quad \psi_{\mathrm{DE} 5}(t):=\log (\exp ((\pi / 2) \sinh t)+1) .
\end{aligned}
$$

The formulas (1.3) with $\psi=\psi_{\mathrm{DE} i}(i=1, \ldots, 5)$ are called the DE-Sinc approximation formulas.

The DE transformations were originally proposed for numerical integration by Takahasi and Mori [12, followed by subsequent extensions and generalizations [3]; $\psi_{\text {DE5 }}$ mentioned above was proposed recently in 4. Use of DE transformations in the Sinc approximation is due to Sugihara [8, 10].

On the other hand, use of single exponential (SE) transformations has been advocated by Stenger [5, 6. Formulas (1.3) with SE transformations $\psi$ are called the SE-Sinc approximation formulas, where the explicit forms of the SE transformations as well as the SE-Sinc formulas are given in Section 2 Historically, the SE-Sinc approximation formulas preceded the DE-Sinc formulas by twenty years.

It is understood in general terms that the SE-Sinc formulas are applicable to larger classes of functions than the DE-Sinc formulas, whereas the DE-Sinc formulas are more efficient for well-behaved functions. Rigorous error analysis has been done for the SE-Sinc formulas and certain classes of functions suited to the SE-Sinc formulas have been identified by Stenger [6]. For the DE-Sinc formulas, on the other hand, Sugihara [8, 10] made an error analysis that led to an observation that the DE-Sinc formulas are nearly optimal in a certain mathematical sense. It must be said, however, that no theorems exist that describe precisely those function classes for which the DE-Sinc formulas are successful.

The first objective of this paper is to identify the function classes suited to the DE-Sinc formulas in a way compatible with the existing results for the SESinc formulas. The DE-Sinc formulas are applicable to more restricted classes of functions, but more efficient for such functions. It may be said that the essence of the present results is already implicit in [8, 10, and the contribution of this paper is to tailor the implicit observation there to explicit statements that are compatible with the corresponding results for the SE-Sinc formulas.

Our theorems for DE-Sinc formulas, as well as the existing theorems of Stenger for SE-Sinc formulas, involve some conditions that are not convenient to verify from a practical point of view. To be more specific, the theorems require certain estimates 
of the function $f$ over complex regions, although approximations are sought on real intervals. To make the theoretical analysis more useful in applications, we present another set of theorems that describe alternative function classes for the DE-Sinc formulas, as well as for the SE-Sinc formulas. The point is that the theorems do not involve upper bound conditions over complex regions but refer only to conditions on the real intervals on which the approximations of $f$ are considered.

Thus the objective of this paper is twofold:

(1) To identify function classes for DE-Sinc formulas in parallel to Stenger's results for SE-Sinc formulas.

(2) To relax the conditions for easier verification, both for DE-Sinc formulas and for SE-Sinc formulas.

This paper is organized as follows. In Section 2, we review Stenger's theorems for the SE-Sinc formulas by way of comparison with our results. In Section 3 , we present our theorems of error estimates for the DE-Sinc formulas as the main result of this paper. Similar error estimates for the DE-Sinc and SE-Sinc formulas are derived under weaker assumptions in Section 4, Sections 5, 6, and 7 are devoted to the proofs.

\section{Function Classes for SUCCESSFul SE-Sinc APproximations}

This section is a review of some relevant results on the approximation formulas based on single exponential transformations.

The single exponential transformations are given by the following functions:

$$
\begin{aligned}
& \psi_{\mathrm{SE} 1}:(-\infty, \infty) \rightarrow(-1,1), \quad \psi_{\mathrm{SE} 1}(t):=\tanh (t / 2), \\
& \psi_{\mathrm{SE} 2}:(-\infty, \infty) \rightarrow(-\infty, \infty), \quad \psi_{\mathrm{SE} 2}(t):=\sinh t, \\
& \psi_{\mathrm{SE} 3}:(-\infty, \infty) \rightarrow(0, \infty), \quad \psi_{\mathrm{SE} 3}(t):=\exp t, \\
& \psi_{\mathrm{SE} 4}:(-\infty, \infty) \rightarrow(0, \infty), \quad \psi_{\mathrm{SE} 4}(t):=\operatorname{arcsinh}(\exp t) .
\end{aligned}
$$

The formulas (1.3) with $\psi=\psi_{\mathrm{SE} i}(i=1, \ldots, 4)$ are called the SE-Sinc approximation formulas.

In the theorems below, functions suited to the SE-Sinc formulas are specified with reference to complex regions. For $d>0$ we define a strip region $\mathcal{D}_{d}$ as

$$
\mathcal{D}_{d}:=\{z \in \mathbf{C}|| \operatorname{Im} z \mid<d\} .
$$

Then we define $\mathcal{D}_{\mathrm{SE} i}(d)$ as the image of $\mathcal{D}_{d}$ through $\psi_{\mathrm{SE} i}$; that is,

$$
\mathcal{D}_{\mathrm{SE} i}(d):=\left\{z=\psi_{\mathrm{SE} i}(w) \mid w \in \mathcal{D}_{d}\right\} \quad(i=1, \ldots, 4) .
$$

Figures 1 to 4 illustrate these regions together with their boundaries $\partial \mathcal{D}_{\mathrm{SE} i}(d)$.

Theorems 2.1 to 2.4 below give asymptotic error estimates for the SE-Sinc formulas with mathematical rigor.

Theorem 2.1 (Stenger [6]). Assume that $f$ is holomorphic on $\mathcal{D}_{\mathrm{SE} 1}(d)$ for $d$ with $0<d<\pi$ and satisfies

$$
\forall z \in \mathcal{D}_{\mathrm{SE} 1}(d):|f(z)| \leq C_{1}\left|\left(1-z^{2}\right)^{\beta}\right|
$$


for constants $C_{1}>0$ and $\beta>0$. Then there exists a constant $C$, independent of $N$, such that

$$
\sup _{-1<x<1}\left|f(x)-\sum_{k=-N}^{N} f\left(\psi_{\mathrm{SE} 1}(k h)\right) S(k, h)\left(\psi_{\mathrm{SE} 1}^{-1}(x)\right)\right| \leq C \sqrt{N} \exp (-\sqrt{\pi d \beta N}),
$$

where $h=\sqrt{\pi d /(\beta N)}$.
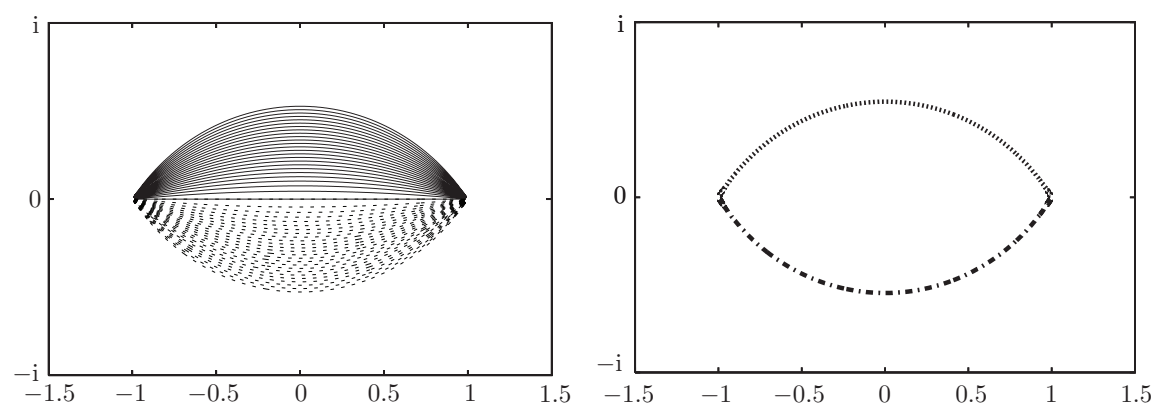

FIGURE 1. Region $\mathcal{D}_{\mathrm{SE} 1}(1)$ and its boundary $\partial \mathcal{D}_{\mathrm{SE} 1}(1)$
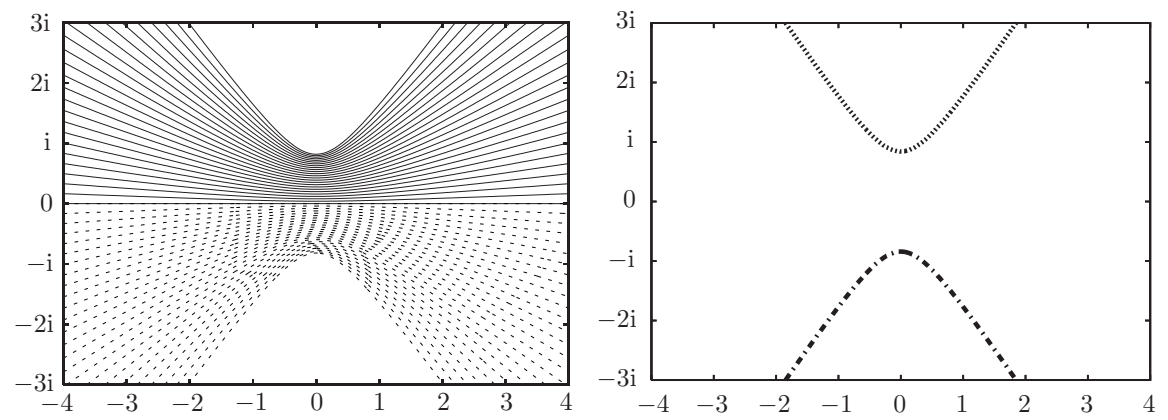

FIGURE 2. Region $\mathcal{D}_{\mathrm{SE} 2}(1)$ and its boundary $\partial \mathcal{D}_{\mathrm{SE} 2}(1)$
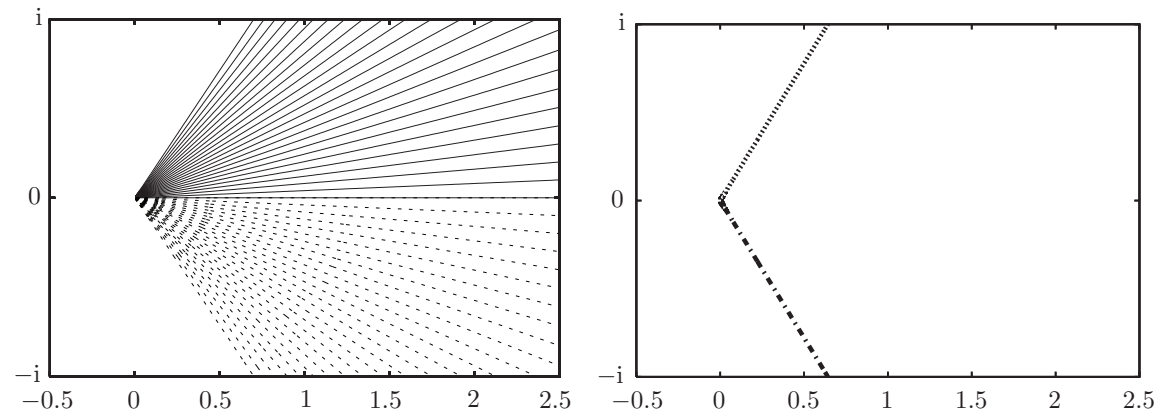

FiguRE 3. Region $\mathcal{D}_{\mathrm{SE} 3}(1)$ and its boundary $\partial \mathcal{D}_{\mathrm{SE} 3}(1)$ 

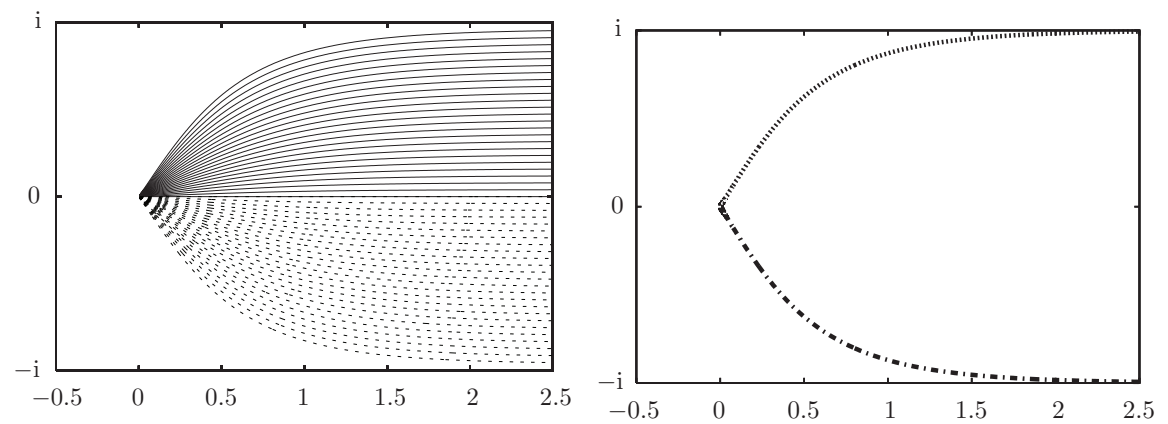

FIGURE 4 . Region $\mathcal{D}_{\mathrm{SE} 4}(1)$ and its boundary $\partial \mathcal{D}_{\mathrm{SE} 4}(1)$

Theorem 2.2 (Stenger [6]). Assume that $f$ is holomorphic on $\mathcal{D}_{\mathrm{SE} 2}(d)$ for $d$ with $0<d<\pi / 2$ and satisfies

$$
\forall z \in \mathcal{D}_{\mathrm{SE} 2}(d):|f(z)| \leq C_{1}\left|\frac{1}{\left(1+z^{2}\right)^{\beta / 2}}\right|
$$

for constants $C_{1}>0$ and $\beta>0$. Then there exists a constant $C$, independent of $N$, such that

$$
\sup _{-\infty<x<\infty}\left|f(x)-\sum_{k=-N}^{N} f\left(\psi_{\mathrm{SE} 2}(k h)\right) S(k, h)\left(\psi_{\mathrm{SE} 2}{ }^{-1}(x)\right)\right| \leq C \sqrt{N} \exp (-\sqrt{\pi d \beta N}),
$$

where $h=\sqrt{\pi d /(\beta N)}$.

Theorem 2.3 (Stenger [6]). Assume that $f$ is holomorphic on $\mathcal{D}_{\mathrm{SE} 3}(d)$ for $d$ with $0<d<\pi / 2$ and satisfies

$$
\forall z \in \mathcal{D}_{\mathrm{SE} 3}(d):|f(z)| \leq C_{1}\left|\frac{z^{\beta}}{\left(1+z^{2}\right)^{\beta}}\right|
$$

for constants $C_{1}>0$ and $\beta>0$. Then there exists a constant $C$, independent of $N$, such that

$$
\sup _{0<x<\infty}\left|f(x)-\sum_{k=-N}^{N} f\left(\psi_{\mathrm{SE} 3}(k h)\right) S(k, h)\left(\psi_{\mathrm{SE} 3}{ }^{-1}(x)\right)\right| \leq C \sqrt{N} \exp (-\sqrt{\pi d \beta N}),
$$

where $h=\sqrt{\pi d /(\beta N)}$.

Theorem 2.4 (Stenger [6]). Assume that $f$ is holomorphic on $\mathcal{D}_{\mathrm{SE} 4}(d)$ for $d$ with $0<d<\pi / 2$ and satisfies

$$
\forall z \in \mathcal{D}_{\mathrm{SE} 4}(d):|f(z)| \leq C_{1}\left|\left(\frac{z}{1+z}\right)^{\beta} \exp (-\beta z)\right|
$$

for constants $C_{1}>0$ and $\beta>0$. Then there exists a constant $C$, independent of $N$, such that

$$
\sup _{0<x<\infty}\left|f(x)-\sum_{k=-N}^{N} f\left(\psi_{\mathrm{SE} 4}(k h)\right) S(k, h)\left(\psi_{\mathrm{SE} 4}{ }^{-1}(x)\right)\right| \leq C \sqrt{N} \exp (-\sqrt{\pi d \beta N}),
$$

where $h=\sqrt{\pi d /(\beta N)}$. 


\section{Function Classes for SuCCESsful DE-SinC APproximations}

In this section, we present our theorems for the error estimate of the DE-Sinc formulas. Recall the transformation functions $\psi_{\mathrm{DE} i}(i=1, \ldots, 5)$ in (1.4)-(1.8):

$$
\begin{aligned}
& \psi_{\mathrm{DE} 1}:(-\infty, \infty) \rightarrow(-1,1), \quad \psi_{\mathrm{DE} 1}(t):=\tanh ((\pi / 2) \sinh t), \\
& \psi_{\mathrm{DE} 2}:(-\infty, \infty) \rightarrow(-\infty, \infty), \quad \psi_{\mathrm{DE} 2}(t):=\sinh ((\pi / 2) \sinh t), \\
& \psi_{\mathrm{DE} 3}:(-\infty, \infty) \rightarrow(0, \infty), \quad \psi_{\mathrm{DE} 3}(t):=\exp ((\pi / 2) \sinh t), \\
& \psi_{\mathrm{DE} 4}:(-\infty, \infty) \rightarrow(0, \infty), \quad \psi_{\mathrm{DE} 4}(t):=\exp (t-\exp (-t)), \\
& \psi_{\mathrm{DE} 5}:(-\infty, \infty) \rightarrow(0, \infty), \quad \psi_{\mathrm{DE} 5}(t):=\log (\exp ((\pi / 2) \sinh t)+1) .
\end{aligned}
$$

To state our theorems we need to introduce complex regions, $\mathcal{D}_{\mathrm{DE} i}(d)$, that are defined as the images of $\mathcal{D}_{d}$ in 2.5 through the transformation functions $\psi_{\mathrm{DE} i}$; that is,

$$
\mathcal{D}_{\mathrm{DE} i}(d):=\left\{z=\psi_{\mathrm{DE} i}(w) \mid w \in \mathcal{D}_{d}\right\} \quad(i=1, \ldots, 5) .
$$

Figures 5 to 9 illustrate these regions together with their boundaries $\partial \mathcal{D}_{\mathrm{DE} i}(d)$. We regard $\mathcal{D}_{\mathrm{DE} i}(d)$ as a region on the Riemann surface.

We are now in the position to state the main theorems. The proofs are shown in Section 5 .
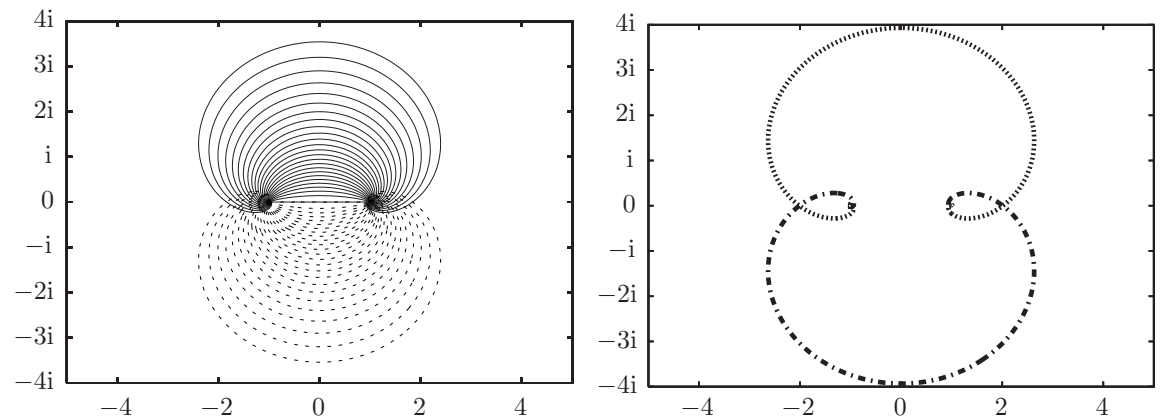

Figure 5. Region $\mathcal{D}_{\mathrm{DE} 1}(1)$ and its boundary $\partial \mathcal{D}_{\mathrm{DE} 1}(1)$
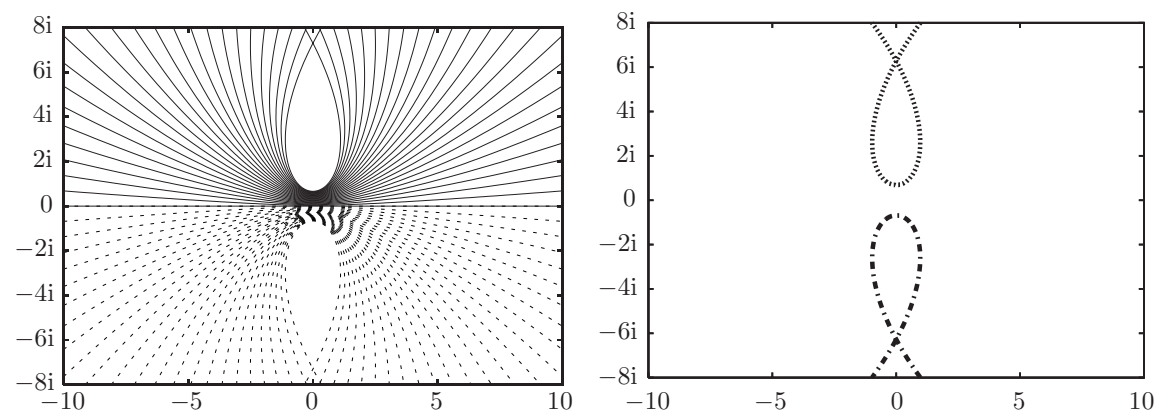

Figure 6 . Region $\mathcal{D}_{\mathrm{DE} 2}(1 / 2)$ and its boundary $\partial \mathcal{D}_{\mathrm{DE} 2}(1 / 2)$ 

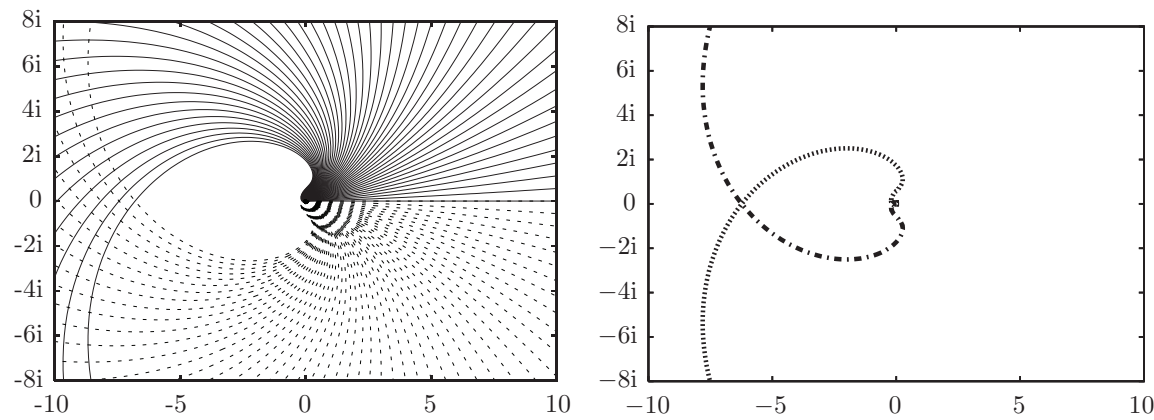

Figure 7 . Region $\mathcal{D}_{\mathrm{DE} 3}(1)$ and its boundary $\partial \mathcal{D}_{\mathrm{DE} 3}(1)$
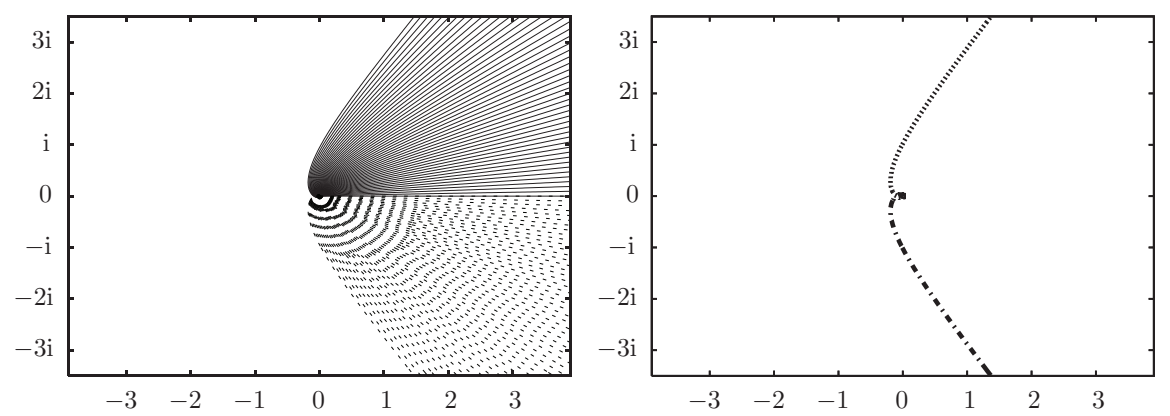

FIGURE 8 . Region $\mathcal{D}_{\mathrm{DE} 4}(1)$ and its boundary $\partial \mathcal{D}_{\mathrm{DE} 4}(1)$
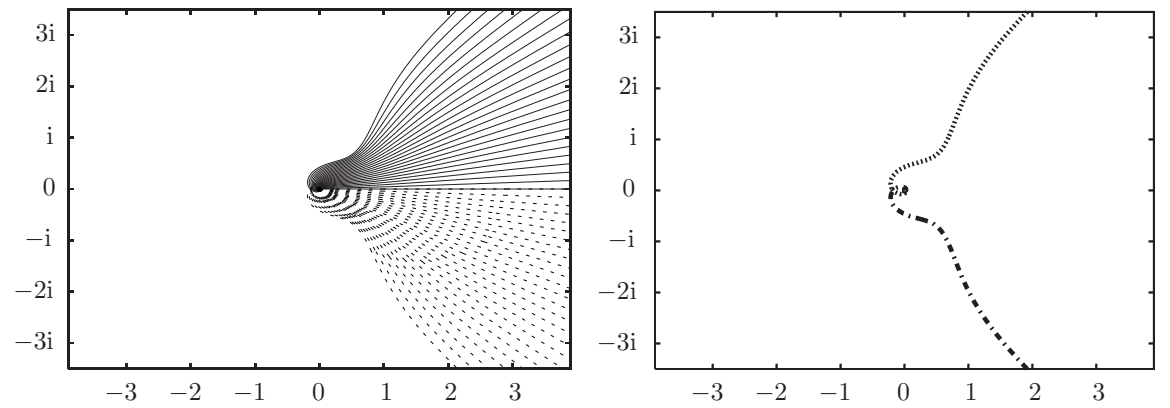

FIGURE 9 . Region $\mathcal{D}_{\mathrm{DE} 5}(1)$ and its boundary $\partial \mathcal{D}_{\mathrm{DE} 5}(1)$

Theorem 3.1. Assume that $f$ is holomorphic on $\mathcal{D}_{\mathrm{DE} 1}(d)$ for $d$ with $0<d<\pi / 2$ and satisfies

$$
\forall z \in \mathcal{D}_{\mathrm{DE} 1}(d):|f(z)| \leq C_{1}\left|\left(1-z^{2}\right)^{\beta}\right|
$$

for constants $C_{1}>0$ and $\beta>0$. Then there exists a constant $C$, independent of $N$, such that

$$
\sup _{-1<x<1}\left|f(x)-\sum_{k=-N}^{N} f\left(\psi_{\mathrm{DE} 1}(k h)\right) S(k, h)\left(\psi_{\mathrm{DE} 1}{ }^{-1}(x)\right)\right| \leq C \exp \left(-\frac{\pi d N}{\log (2 d N / \beta)}\right),
$$

where $h=(\log (2 d N / \beta)) / N$. 
Theorem 3.2. Assume that $f$ is holomorphic on $\mathcal{D}_{\mathrm{DE} 2}(d)$ for $d$ with $0<d<\pi / 2$ and satisfies

$$
\forall z \in \mathcal{D}_{\mathrm{DE} 2}(d):|f(z)| \leq C_{1}\left|\frac{1}{\left(1+z^{2}\right)^{\beta / 2}}\right|
$$

for constants $C_{1}>0$ and $\beta>0$. Then there exists a constant $C$, independent of $N$, such that

$$
\sup _{-\infty<x<\infty}\left|f(x)-\sum_{k=-N}^{N} f\left(\psi_{\mathrm{DE} 2}(k h)\right) S(k, h)\left(\psi_{\mathrm{DE} 2}{ }^{-1}(x)\right)\right| \leq C \exp \left(-\frac{\pi d N}{\log (4 d N / \beta)}\right),
$$

where $h=(\log (4 d N / \beta)) / N$.

Theorem 3.3. Assume that $f$ is holomorphic on $\mathcal{D}_{\mathrm{DE} 3}(d)$ for $d$ with $0<d<\pi / 2$ and satisfies

$$
\forall z \in \mathcal{D}_{\mathrm{DE} 3}(d):|f(z)| \leq C_{1}\left|\frac{z^{\beta}}{\left(1+z^{2}\right)^{\beta}}\right|
$$

for constants $C_{1}>0$ and $\beta>0$. Then there exists a constant $C$, independent of $N$, such that

$$
\sup _{0<x<\infty}\left|f(x)-\sum_{k=-N}^{N} f\left(\psi_{\mathrm{DE} 3}(k h)\right) S(k, h)\left(\psi_{\mathrm{DE} 3}{ }^{-1}(x)\right)\right| \leq C \exp \left(-\frac{\pi d N}{\log (4 d N / \beta)}\right),
$$

where $h=(\log (4 d N / \beta)) / N$.

Theorem 3.4. Assume that $f$ is holomorphic on $\mathcal{D}_{\mathrm{DE} 4}(d)$ for $d$ with $0<d<\pi / 2$ and satisfies

$$
\forall z \in \mathcal{D}_{\mathrm{DE} 4}(d):|f(z)| \leq C_{1}\left|\left(\frac{z}{1+z}\right)^{\beta} \exp (-\beta z)\right|
$$

for constants $C_{1}>0$ and $\beta>0$. Then there exists a constant $C$, independent of $N$, such that

$$
\sup _{0<x<\infty}\left|f(x)-\sum_{k=-N}^{N} f\left(\psi_{\mathrm{DE} 4}(k h)\right) S(k, h)\left(\psi_{\mathrm{DE} 4}{ }^{-1}(x)\right)\right| \leq C \exp \left(-\frac{\pi d N}{\log (\pi d N / \beta)}\right),
$$

where $h=(\log (\pi d N / \beta)) / N$.

Theorem 3.5. Assume that $f$ is holomorphic on $\mathcal{D}_{\mathrm{DE} 5}(d)$ for $d$ with $0<d<\pi / 2$ and satisfies

$$
\forall z \in \mathcal{D}_{\mathrm{DE} 5}(d):|f(z)| \leq C_{1}\left|\left(\frac{z}{1+z}\right)^{\beta} \exp (-\beta z)\right|
$$

for constants $C_{1}>0$ and $\beta>0$. Then there exists a constant $C$, independent of $N$, such that

$$
\sup _{0<x<\infty}\left|f(x)-\sum_{k=-N}^{N} f\left(\psi_{\mathrm{DE} 5}(k h)\right) S(k, h)\left(\psi_{\mathrm{DE} 5}{ }^{-1}(x)\right)\right| \leq C \exp \left(-\frac{\pi d N}{\log (4 d N / \beta)}\right),
$$

where $h=(\log (4 d N / \beta)) / N$. 


\section{Alternative Classes for DE-SinC AND SE-SinC APproximations}

Our theorems in the previous section involve upper bounds on the behavior of $f$ over the complex regions $\mathcal{D}_{\mathrm{DE} i}(d)$. For instance, Theorem 3.1 imposes the condition in (3.1) that $|f(z)| \leq C_{1}\left|\left(1-z^{2}\right)^{\beta}\right|$ holds for all $z \in \mathcal{D}_{\mathrm{DE} 1}(d)$. Such conditions, however, are difficult to verify in practical situations. It would be nicer if they could be replaced by conditions only on real intervals, such as $|f(x)| \leq C_{1}\left|\left(1-x^{2}\right)^{\beta}\right|$ for all $x \in(-1,1)$.

It is in fact possible to establish theorems for the DE-Sinc formulas that do not involve upper bound conditions over the complex regions $\mathcal{D}_{\mathrm{DE} i}(d)$, but refer only to conditions on the real intervals on which the approximations of $f$ are considered. To be more precise, such theorems have two types of assumption:1:

(1) $f$ is holomorphic and bounded on $\mathcal{D}_{\mathrm{DE} i}(d)$, and

(2) $f$ is upper bounded by a certain simple function on the real interval,

under which error estimates similar to Theorems 3.1 to 3.5 are obtained.

We state the theorem for $\psi_{\mathrm{DE} 1}(t)$ only, while referring the reader to [14 for the theorems for $\psi_{\mathrm{DEi}}(t)(i=2,3,4,5)$. The theorems for $\psi_{\mathrm{DEi}}(t)(i=2,3,4,5)$ can be obtained easily from Theorems 3.2, 3.3, 3.4 and 3.5, respectively, just as the theorem for $\psi_{\mathrm{DE} 1}(t)$ below is obtained from Theorem 3.1. The proof of the theorem below is given in Section [6]

Theorem 4.1. Assume that $f$ is holomorphic and bounded on $\mathcal{D}_{\mathrm{DE} 1}(d)$ for $d$ with $0<d<\pi / 2$ and satisfies

$$
\forall x \in(-1,1):|f(x)| \leq C_{1}\left|\left(1-x^{2}\right)^{\beta}\right|
$$

for constants $C_{1}>0$ and $\beta>0$. Then, for any $\varepsilon$ with $0<\varepsilon<d$, there exists a constant $C_{\varepsilon}$, independent of $N$, such that

$$
\sup _{-1<x<1}\left|f(x)-\sum_{k=-N}^{N} f\left(\psi_{\mathrm{DE} 1}(k h)\right) S(k, h)\left(\psi_{\mathrm{DE} 1}{ }^{-1}(x)\right)\right| \leq C_{\varepsilon} \exp \left(-\frac{\pi(d-\varepsilon) N}{\log (2 d N / \beta)}\right),
$$

where $h=(\log (2 d N / \beta)) / N$.

Theorems of the same vein can also be established for the SE-Sinc formulas. Such theorems do not involve upper bound conditions over the complex regions $\mathcal{D}_{\mathrm{SE} i}(d)$, but refer only to conditions on the real intervals on which the approximations of $f$ are considered.

Here we show the theorem for $\psi_{\mathrm{SE} 1}(t)$ only for the same reason as above; see 14 for the other cases. The proof of the theorem below is given in Section 7

Theorem 4.2. Assume that $f$ is holomorphic and bounded on $\mathcal{D}_{\mathrm{SE} 1}(d)$ for $d$ with $0<d<\pi$ and satisfies

$$
\forall x \in(-1,1):|f(x)| \leq C_{1}\left|\left(1-x^{2}\right)^{\beta}\right|
$$

\footnotetext{
${ }^{1}$ Boundedness of $f$ is not assumed in Theorems 3.1 to 3.5 because it is implied by each of the conditions 3.1 to 3.5.
} 
for constants $C_{1}>0$ and $\beta>0$. Then, for any $\varepsilon$ with $0<\varepsilon<d$, there exists a constant $C_{\varepsilon}$, independent of $N$, such that

$$
\begin{array}{r}
\sup _{-1<x<1}\left|f(x)-\sum_{k=-N}^{N} f\left(\psi_{\mathrm{SE} 1}(k h)\right) S(k, h)\left(\psi_{\mathrm{SE} 1}{ }^{-1}(x)\right)\right| \\
\leq C_{\varepsilon} \sqrt{N} \exp (-\sqrt{\pi(d-\varepsilon) \beta N}),
\end{array}
$$

where $h=\sqrt{\pi d /(\beta N)}$.

\section{Proofs of Theorems 3.1 to 3.5}

In this section, we prove Theorems 3.1 to 3.5 in turn. The proofs are based on Theorem 5.1 below, which is a well known error estimate for the Sinc formula on $(-\infty, \infty)$ for functions with double exponential decay.

5.1. Fundamental theorems for DE-Sinc formula. As a preliminary we present here the error estimate for the basic Sinc formula on $(-\infty, \infty)$ for functions with double exponential decay. For $\varepsilon$ with $0<\varepsilon<1$, we define

$$
\mathcal{D}_{d}(\varepsilon):=\{z \in \mathbf{C}|| \operatorname{Re} z|<1 / \varepsilon,| \operatorname{Im} z \mid<d(1-\varepsilon)\}
$$

and also

$$
\mathcal{N}_{1}\left(f, \mathcal{D}_{d}\right):=\lim _{\varepsilon \rightarrow 0} \int_{\partial \mathcal{D}_{d}(\varepsilon)}|f(z)||\mathrm{d} z|
$$

for a function $f$ on $\mathcal{D}_{d}$. With these definitions, we introduce a function space

$$
\mathbf{H}^{1}\left(\mathcal{D}_{d}\right):=\left\{f: \mathcal{D}_{d} \rightarrow \mathbf{C} \mid f \text { is holomorphic on } \mathcal{D}_{d} \text { and satisfies } \mathcal{N}_{1}\left(f, \mathcal{D}_{d}\right)<\infty\right\} \text {. }
$$

Theorem 5.1 ([10], [11, Theorem 5]). Assume that a function $f$ satisfies

$$
\begin{aligned}
& f \in \mathbf{H}^{1}\left(\mathcal{D}_{d}\right), \\
& \forall x \in \mathbf{R}:|f(x)| \leq A \exp (-B \exp (\gamma|x|))
\end{aligned}
$$

for positive constants $A, B, \gamma$ and $d$, where $\gamma d \leq \pi / 2$. Then there exists a constant $C$, independent of $N$, such that

$$
\sup _{-\infty<x<\infty}\left|f(x)-\sum_{k=-N}^{N} f(k h) S(k, h)(x)\right| \leq C \exp \left(-\frac{\pi d \gamma N}{\log (\pi d \gamma N / B)}\right),
$$

where

$$
h=\frac{\log (\pi d \gamma N / B)}{\gamma N} .
$$

Proof. A sketch of the proof is given here in view of its fundamental role in subsequent arguments. We divide the error into two parts as

$$
\begin{aligned}
& \sup _{-\infty<x<\infty}\left|f(x)-\sum_{k=-N}^{N} f(k h) S(k, h)(x)\right| \\
& \leq \sup _{-\infty<x<\infty}\left|f(x)-\sum_{k=-\infty}^{\infty} f(k h) S(k, h)(x)\right|+\sup _{-\infty<x<\infty}\left|\sum_{|k|>N} f(k h) S(k, h)(x)\right| .
\end{aligned}
$$


The first term on the right-hand side may be referred to as the sampling error and the second as the truncation error. For the sampling error it follows from (5.1), (5.3), and an estimate by a contour integral that

$$
\begin{aligned}
& \sup _{-\infty<x<\infty}\left|f(x)-\sum_{k=-\infty}^{\infty} f(k h) S(k, h)(x)\right| \\
& \leq C^{\prime} \exp \left(-\frac{\pi d}{h}\right)=C^{\prime} \exp \left(-\frac{\pi d \gamma N}{\log (\pi d \gamma N / B)}\right),
\end{aligned}
$$

where $C^{\prime}>0$ is a constant. For the truncation error we have from (5.2) and (5.3) that

$$
\begin{aligned}
& \sup _{-\infty<x<\infty}\left|\sum_{|k|>N} f(k h) S(k, h)(x)\right| \leq \sum_{|k|>N}|f(k h)| \\
& \leq \frac{2 A \exp (-B \exp (\gamma N h))}{B \gamma \exp (\gamma N h)}=\frac{2 A \exp (-2 \pi d \gamma N)}{2 \pi d \gamma^{2} N} .
\end{aligned}
$$

Hence follows the claim.

The following lemma gives a sufficient condition for $f$ to satisfy the first assumption $f \in \mathbf{H}^{1}\left(\mathcal{D}_{d}\right)$ in Theorem 5.1 in terms of a dominating function $g$ of $f$.

Lemma 5.2. A function $f$ holomorphic on $\mathcal{D}_{d}$ belongs to $\mathbf{H}^{1}\left(\mathcal{D}_{d}\right)$, if there exists a function $g$ on $\overline{\mathcal{D}_{d}}$ such that

$$
\begin{gathered}
\forall z \in \mathcal{D}_{d}:|f(z)| \leq|g(z)|, \\
\forall x \in \mathbf{R}, \forall y \in \mathbf{R}(|y| \leq d):|g(x+\mathrm{i} y)| \leq A^{\prime} \exp \left(-B^{\prime} \exp \left(\gamma^{\prime}|x|\right)\right)
\end{gathered}
$$

for constants $A^{\prime}, B^{\prime}, \gamma^{\prime}>0$.

Proof. By (5.8) we have

$$
\begin{aligned}
& \int_{-\infty}^{\infty}(|g(x+\mathrm{i} d)|+|g(x-\mathrm{i} d)|) \mathrm{d} x<\infty, \\
& \lim _{x \rightarrow \pm \infty} \int_{-d}^{d}|g(x+\mathrm{i} y)| \mathrm{d} y=0 .
\end{aligned}
$$

Then, by (5.7), we see that $\mathcal{N}_{1}\left(f, \mathcal{D}_{d}\right) \leq \mathcal{N}_{1}\left(g, \mathcal{D}_{d}\right)<\infty$.

Some comment is in order on the inequality $\gamma d \leq \pi / 2$ in Theorem 5.1. This inequality condition is natural and inevitable because of the following fact, which is an immediate corollary of [9, Lemma 4.2].

Theorem 5.3 (Vanishing Theorem). Let $A$ and $B$ be positive constants, and let $\gamma$ and $d$ be positive constants with $\gamma d>\pi / 2$. If a function $f$ on $\mathcal{D}_{d}$ satisfies

$$
\begin{aligned}
& f \in \mathbf{H}^{1}\left(\mathcal{D}_{d}\right), \\
& \forall x \in \mathbf{R}:|f(x)| \leq A \exp (-B \exp (\gamma|x|)),
\end{aligned}
$$

then $f \equiv 0$.

It should be clear that the above theorem does not affect the proofs of the theorems and lemmas in this paper. 
5.2. Proof of Theorem 3.1. In the proofs of Theorem 3.1 to 3.5 in Subsections 5.2 to [5.6. we show that the transformed function $\tilde{f}=f\left(\psi_{\mathrm{DE} i}(\cdot)\right)$ satisfies the assumptions of Theorem 5.1 by demonstrating a dominating function $g$ for $\tilde{f}$ as described in Lemma 5.2.

In this subsection we deal with $\psi_{\mathrm{DE} 1}$. The transformed function $\tilde{f}=f\left(\psi_{\mathrm{DE} 1}(\cdot)\right)$ is holomorphic on $\mathcal{D}_{d}$. Since

$$
\forall z \in \mathcal{D}_{d}:\left|f\left(\psi_{\mathrm{DE} 1}(z)\right)\right| \leq C_{1}\left|\frac{1}{\left\{\cosh ^{2}((\pi / 2) \sinh z)\right\}^{\beta}}\right|
$$

follows from (3.1), we can take

$$
g(z)=C_{1} \frac{1}{\left\{\cosh ^{2}((\pi / 2) \sinh z)\right\}^{\beta}}
$$

to meet the first requirement (5.7) in Lemma 5.2. We can also show that this function $g(z)$ satisfies (5.8) by letting $B=\beta$ in Lemma 5.4 below. Therefore, we have $f\left(\psi_{\mathrm{DE} 1}(\cdot)\right) \in \mathbf{H}^{1}\left(\mathcal{D}_{d}\right)$ by Lemma 5.2. For the condition (5.2) in Theorem 5.1. it follows from the above inequality that, for $x \in \mathbf{R}$, we have

$$
\left|f\left(\psi_{\mathrm{DE} 1}(x)\right)\right| \leq A \exp \left(-\frac{\pi \beta}{2} \exp (|x|)\right)
$$

for a constant $A>0$. Therefore, $f\left(\psi_{\mathrm{DE} 1}(\cdot)\right)$ satisfies the assumptions of Theorem 5.1 for $B=\pi \beta / 2$ and $\gamma=1$. Hence follows the claim of Theorem 3.1 .

Lemma 5.4. Let $d$ be a constant with $0<d<\pi / 2$, and $B$ be a positive constant. Then the function $g(z):=1 /\left\{\cosh ^{2}((\pi / 2) \sinh z)\right\}^{B}$ satisfies (5.8).

Proof. Let $x, y \in \mathbf{R}$ and $|y| \leq d$. We have

$$
\begin{aligned}
& |\cosh ((\pi / 2) \sinh (x+\mathrm{i} y))|^{2} \\
& =\cosh ^{2}((\pi / 2) \sinh x \cos y)-\sin ^{2}((\pi / 2) \cosh x \sin y) \\
& \geq \cosh ^{2}(((\pi / 2) \cos d) \sinh x)-\sin ^{2}((\pi / 2) \cosh x \sin y) \\
& \geq \begin{cases}1-\sin ^{2}((\pi / 2) \cosh \delta \sin d)=1 / 2 & (|x| \leq \delta), \\
\cosh ^{2}(((\pi / 2) \cos d) \sinh x)-1=\sinh ^{2}(((\pi / 2) \cos d) \sinh x) & (|x|>\delta),\end{cases}
\end{aligned}
$$

where $\delta=\operatorname{arccosh}(1 /(2 \sin d))$. Hence

$$
|g(x+\mathrm{i} y)| \leq \begin{cases}2^{2 B} & (|x| \leq \delta) \\ 1 /[\sinh (((\pi / 2) \cos d) \sinh x)]^{2 B} & (|x|>\delta)\end{cases}
$$

5.3. Proof of Theorem 3.2. First, the transformed function $f\left(\psi_{\mathrm{DE} 2}(\cdot)\right)$ is holomorphic on $\mathcal{D}_{d}$. By (3.2), we have

$$
\forall z \in \mathcal{D}_{d}:\left|f\left(\psi_{\mathrm{DE} 2}(z)\right)\right| \leq C_{1}\left|\frac{1}{\left\{\cosh ^{2}((\pi / 2) \sinh z)\right\}^{\beta / 2}}\right| .
$$

Then the rest of the proof is similar to that of Theorem 3.1. Note that we set $B=\beta / 2$ in Lemma 5.4 to show (5.8). 
5.4. Proof of Theorem 3.3. First, the transformed function $f\left(\psi_{\mathrm{DE} 3}(\cdot)\right)$ is holomorphic on $\mathcal{D}_{d}$. By (3.3), we have

$$
\forall z \in \mathcal{D}_{d}:\left|f\left(\psi_{\mathrm{DE} 3}(z)\right)\right| \leq \frac{C_{1}}{2^{\beta}}\left|\frac{1}{\left\{\cosh ^{2}((\pi / 2) \sinh z)\right\}^{\beta / 2}}\right| .
$$

Then the rest of the proof is similar to that of Theorem 3.1. Note that we set $B=\beta / 2$ in Lemma 5.4 to show (5.8).

5.5. Proof of Theorem 3.4. First, the transformed function $f\left(\psi_{\mathrm{DE} 4}(\cdot)\right)$ is holomorphic on $\mathcal{D}_{d}$. It follows from (3.4) that

$$
\begin{aligned}
& \forall z \in \mathcal{D}_{d}: \\
& \left|f\left(\psi_{\mathrm{DE} 4}(z)\right)\right| \leq C_{1}\left|\left\{\frac{\exp z}{\exp z+\exp (\exp (-z))}\right\}^{\beta} \exp (-\beta \exp z \cdot \exp (-\exp (-z)))\right| .
\end{aligned}
$$

Accordingly, we choose the right-hand side above as $g(z)$ in Lemma 5.2. Then (5.7) is met. This function $g(z)$ also satisfies (5.8). Therefore, we have $f\left(\psi_{\mathrm{DE} 4}(\cdot)\right) \in$ $\mathbf{H}^{1}\left(\mathcal{D}_{d}\right)$ by Lemma 5.2 As for the other condition (5.2) in Theorem 5.1, it follows from the above inequality that, for $x \in \mathbf{R}$ we have

$$
\left|f\left(\psi_{\mathrm{DE} 4}(x)\right)\right| \leq A \exp (-\beta \exp (|x|))
$$

for a constant $A>0$. Thus $f\left(\psi_{\mathrm{DE} 4}(\cdot)\right)$ satisfies the assumptions of Theorem 5.1 for $B=\beta$ and $\gamma=1$. Hence follows the claim of Theorem 3.4

5.6. Proof of Theorem 3.5. First, the transformed function $f\left(\psi_{\mathrm{DE} 5}(\cdot)\right)$ is holomorphic on $\mathcal{D}_{d}$. Since

$$
\begin{aligned}
\forall z \in \mathcal{D}_{d}:\left|f\left(\psi_{\mathrm{DE} 5}(z)\right)\right| \leq C_{1} \mid & \left(\frac{\log (\exp ((\pi / 2) \sinh z)+1)}{1+\log (\exp ((\pi / 2) \sinh z)+1)}\right)^{\beta} \\
& \cdot \exp (-\beta \log (\exp ((\pi / 2) \sinh z)+1)) \mid
\end{aligned}
$$

by (3.5), we can choose the right-hand side above as $g(z)$ in Lemma 5.2. Then (5.7) is met. This function $g(z)$ also satisfies (5.8). Therefore, we have $f\left(\psi_{\operatorname{DE} 5}(\cdot)\right) \in$ $\mathbf{H}^{1}\left(\mathcal{D}_{d}\right)$ by Lemma 5.2. As for the second condition (5.2) in Theorem 5.1] it can be shown that

$$
\left|f\left(\psi_{\mathrm{DE} 5}(x)\right)\right| \leq A \exp \left(-\frac{\pi \beta}{4} \exp (|x|)\right)
$$

holds for $x \in \mathbf{R}$ with a constant $A>0$. Therefore, $f\left(\psi_{\text {DE5 }}(\cdot)\right)$ satisfies the assumptions of Theorem 5.1 for $B=\pi \beta / 4$ and $\gamma=1$. Thus we have proven Theorem 3.5 .

\section{Proof of Theorem 4.1}

To cope with the weaker decay condition in Theorem 4.1 we first modify the fundamental theorem (Theorem 5.1) for the Sinc formula on $(-\infty, \infty)$. To be specific, we relax the assumption by replacing the requirement of $f \in \mathbf{H}^{1}\left(\mathcal{D}_{d}\right)$ in (5.1) with the condition that $f$ is holomorphic and bounded on $\mathcal{D}_{d}$. The resulting theorem (Theorem 6.2), giving almost the same error estimate under milder conditions, will serve as the basis of our proof, just as Theorem 5.1 did for Theorem 3.1; see Table 1

The following is a key lemma. 
TABLE 1. Fundamental theorems for approximation on $(-\infty, \infty)$

DE-Sinc formula

\begin{tabular}{rcccc}
\hline Theorem 5.1 & Theorem 6.2 & & Theorem 7.1 & Theorem 7.2 \\
$f \in \mathbf{H}^{1}\left(\mathcal{D}_{d}\right)$ & hol/bnd on $\mathcal{D}_{d}$ & & $f \in \mathbf{H}^{1}\left(\mathcal{D}_{d}\right)$ & hol/bnd on $\mathcal{D}_{d}$ \\
double exponential decay on $\mathbf{R}$ & & single exponential decay on $\mathbf{R}$ \\
\hline "hol/bnd" & $=$ "holomorphic and bounded")
\end{tabular}

Lemma 6.1 ([13, Lemma 5.5]). Assume that a function $f$ is holomorphic and bounded on $\overline{\mathcal{D}_{d}}$ for $d>0$, and it satisfies

$$
\forall x \in \mathbf{R}:|f(x)| \leq A \exp (-B \exp (\gamma|x|))
$$

for constants $A, B>0$, and $\gamma>0$ with $\gamma d \leq \pi / 2$. Then there exists a constant $M_{d}$ such that

$\forall x \in \mathbf{R}, \forall y \in \mathbf{R}(|y|<d):|f(x+\mathrm{i} y)| \leq M_{d} \exp \left(-B \frac{\sin (\gamma(d-|y|))}{\sin (\gamma d)} \exp (\gamma|x|)\right)$.

With this lemma, we can show the following.

Theorem 6.2. Assume that a function $f$ is holomorphic and bounded on $\mathcal{D}_{d}$ for $d>0$, and it satisfies

$$
\forall x \in \mathbf{R}:|f(x)| \leq A \exp (-B \exp (\gamma|x|))
$$

for constants $A, B>0$ and $\gamma>0$ with $\gamma d \leq \pi / 2$. Then, for arbitrary $\varepsilon$ with $0<\varepsilon<d$, there exists a constant $C_{\varepsilon}$, independent of $N$, such that

$$
\sup _{-\infty<x<\infty}\left|f(x)-\sum_{k=-N}^{N} f(k h) S(k, h)(x)\right| \leq C_{\varepsilon} \exp \left(-\frac{\pi(d-\varepsilon) \gamma N}{\log (\pi d \gamma N / B)}\right),
$$

where $h=(\log (\pi d \gamma N / B)) /(\gamma N)$.

Proof. By the assumption, $f$ is holomorphic and bounded on $\overline{\mathcal{D}_{d-\varepsilon / 2}}$. It then follows from Lemma 6.1 that

$$
\forall z \in \overline{\mathcal{D}_{d-\varepsilon}}:|f(z)| \leq M_{d-\varepsilon / 2} \exp \left(-B \frac{\sin (\gamma \varepsilon / 2)}{\sin (\gamma(d-\varepsilon / 2))} \exp (\gamma|\operatorname{Re} z|)\right)
$$

for some $M_{d-\varepsilon / 2}$. This implies $f \in \mathbf{H}^{1}\left(\mathcal{D}_{d-\varepsilon}\right)$ by Lemma 5.2

The rest of the proof is similar to that of Theorem 5.1. Just as (5.5) we have

$$
\begin{aligned}
& \sup _{-\infty<x<\infty}\left|f(x)-\sum_{k=-\infty}^{\infty} f(k h) S(k, h)(x)\right| \\
& \leq C_{\varepsilon}^{\prime} \exp \left(-\frac{\pi(d-\varepsilon)}{h}\right)=C_{\varepsilon}^{\prime} \exp \left(-\frac{\pi(d-\varepsilon) \gamma N}{\log (\pi d \gamma N / B)}\right)
\end{aligned}
$$

for the sampling error. For the truncation error we have

$$
\sup _{-\infty<x<\infty}\left|\sum_{|k|>N} f(k h) S(k, h)(x)\right| \leq \frac{2 A \exp (-2 \pi d \gamma N)}{2 \pi d \gamma^{2} N},
$$

the same estimate as in (5.6). Hence follows (6.2). 
Proof of Theorem 4.1. The transformed function $\tilde{f}=f\left(\psi_{\mathrm{DE} 1}(\cdot)\right)$ is holomorphic and bounded on $\mathcal{D}_{d}$. For the condition (6.1) we have

$$
\left|f\left(\psi_{\mathrm{DE} 1}(x)\right)\right| \leq A \exp \left(-\frac{\pi \beta}{2} \exp (|x|)\right)
$$

for $x \in \mathbf{R}$ with a constant $A>0$. Therefore, $\tilde{f}=f\left(\psi_{\mathrm{DE} 1}(\cdot)\right)$ satisfies the assumptions of Theorem 6.2 for $B=\pi \beta / 2$ and $\gamma=1$. Hence follows the claim.

\section{Proof of Theorem 4.2}

We mention here two fundamental theorems for the Sinc formula on $(-\infty, \infty)$ for functions with single exponential decay. The first is a well-known fact due to Stenger and the second is a similar statement under a weaker assumption; see also Table 1 .

Theorem 7.1 (Stenger [6]). Assume that a function $f$ satisfies

$$
\begin{aligned}
& f \in \mathbf{H}^{1}\left(\mathcal{D}_{d}\right), \\
& \forall x \in \mathbf{R}:|f(x)| \leq \alpha \exp (-\beta|x|)
\end{aligned}
$$

for positive constants $\alpha, \beta$, and $d$. Then there exists a constant $C$, independent of $N$, such that

$$
\sup _{-\infty<x<\infty}\left|f(x)-\sum_{k=-N}^{N} f(k h) S(k, h)(x)\right| \leq C \sqrt{N} \exp (-\sqrt{\pi d \beta N}),
$$

where $h=\sqrt{\pi d /(\beta N)}$.

Proof. A sketch of the proof is given here in view of its fundamental role in subsequent arguments. Recall (5.4). By the assumption (7.1), the sampling error is estimated as

$$
\sup _{-\infty<x<\infty}\left|f(x)-\sum_{k=-\infty}^{\infty} f(k h) S(k, h)(x)\right| \leq C^{\prime} \exp \left(-\frac{\pi d}{h}\right)=C^{\prime} \exp (-\sqrt{\pi d \beta N})
$$

for a constant $C^{\prime}$. By (17.2), on the other hand, the truncation error is estimated as

$$
\begin{aligned}
& \sup _{-\infty<x<\infty}\left|\sum_{|k|>N} f(k h) S(k, h)(x)\right| \leq \sum_{|k|>N}|f(k h)| \\
& \leq \frac{2 \alpha}{\beta h} \exp (-\beta N h)=\frac{2 \alpha \sqrt{N}}{\sqrt{\pi \beta d}} \exp (-\sqrt{\pi d \beta N}) .
\end{aligned}
$$

Hence follows (7.3).

To cope with the weaker decay condition in Theorem 4.2, we need to modify the fundamental theorem above to the following form.

Theorem 7.2. Assume that a function $f$ is holomorphic and bounded on $\mathcal{D}_{d}$ for $d>0$, and it satisfies

$$
\forall x \in \mathbf{R}:|f(x)| \leq \alpha \exp (-\beta|x|)
$$




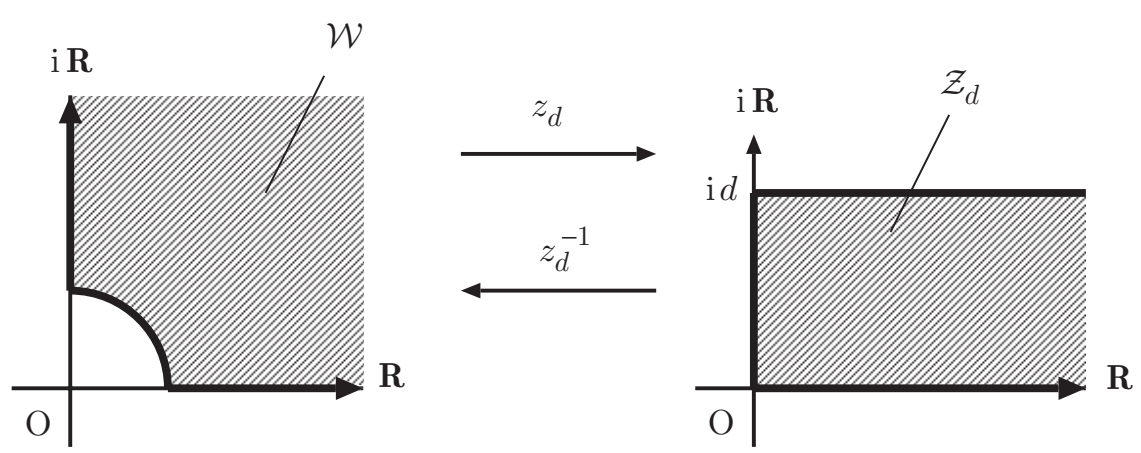

Figure $10 . \mathcal{W}$ and $\mathcal{Z}_{d}$

for positive constants $\alpha$ and $\beta$. Then, for arbitrary $\varepsilon$ with $0<\varepsilon<d$, there exists a constant $C_{\varepsilon}$, independent of $N$, such that

$$
\sup _{-\infty<x<\infty}\left|f(x)-\sum_{k=-N}^{N} f(k h) S(k, h)(x)\right| \leq C_{\varepsilon} \sqrt{N} \exp (-\sqrt{\pi(d-\varepsilon) \beta N}),
$$

where $h=\sqrt{\pi d /(\beta N)}$.

Proof. The proof is given later.

Proof of Theorem 4.2, The transformed function $f\left(\psi_{\mathrm{SE} 1}(\cdot)\right)$ is holomorphic and bounded on $\mathcal{D}_{d}$. For the condition (7.6) we have

$$
\left|f\left(\psi_{\mathrm{SE} 1}(x)\right)\right| \leq \alpha \exp (-\beta|x|)
$$

for $x \in \mathbf{R}$ with a constant $\alpha>0$. Therefore, $f\left(\psi_{\mathrm{SE} 1}(\cdot)\right)$ satisfies the assumptions of Theorem 7.2. Hence follows the claim.

Finally, we prove Theorem 7.2 by establishing a new lemma (Lemma 7.5), which plays the role of Lemma 6.1 in the proof of Theorem 6.2.

We start with the following theorem, a variant of the Phragmén-Lindelöf theorem 1. Theorem 1.4.1]. As in Figure 10 (left) we define a complex region $\mathcal{W}$ as

$$
\mathcal{W}:=\left\{x+\mathrm{i} y \mid x, y \in \mathbf{R}, x, y>0, x^{2}+y^{2}>1\right\} .
$$

Theorem 7.3. Assume that a function $f: \overline{\mathcal{W}} \rightarrow \mathbf{C}$ is holomorphic on $\mathcal{W}$ and continuous on $\overline{\mathcal{W}}$. Also assume that

$$
\forall w \in \partial \mathcal{W}:|f(w)| \leq M
$$

for a constant $M>0$. If there exists a real number $\rho<2$ such that

$$
\left|f\left(r \mathrm{e}^{\mathrm{i} \theta}\right)\right|=\mathrm{O}\left(\exp \left(r^{\rho}\right)\right) \quad(r \rightarrow \infty)
$$

holds uniformly with respect to $\theta \in(0, \pi / 2)$, then we have

$$
\forall w \in \mathcal{W}:|f(w)| \leq M .
$$

Proof. The proof is similar to that of [1, Theorem 1.4.1], and is omitted here. 
For $d>0$ define a complex region $\mathcal{Z}_{d}$ (see Figure 10) as

$$
\mathcal{Z}_{d}:=\{x+\mathrm{i} y \mid x, y \in \mathbf{R}, x>0,0<y<d\}
$$

and a mapping $z_{d}: \mathcal{W} \rightarrow \mathcal{Z}_{d}$ as

$$
z_{d}(w):=\frac{2 d}{\pi} \log w
$$

where the logarithm is considered on $\mathbf{C} \backslash(-\infty, 0]$ with the argument in $(-\pi, \pi)$.

By translating Theorem 7.3 for $\mathcal{W}$ to a statement for $\mathcal{Z}_{d}$ through the mapping $z_{d}$ we obtain the following.

Corollary 7.4. For $d>0$ assume that a function $f: \overline{\mathcal{Z}_{d}} \rightarrow \mathbf{C}$ is holomorphic on $\mathcal{Z}_{d}$ and continuous on $\overline{\mathcal{Z}_{d}}$, and that

$$
\forall z \in \partial \mathcal{Z}_{d}:|f(z)| \leq M
$$

for a constant $M>0$. If there exists a real number $\rho<2$ such that

$$
|f(x+\mathrm{i} y)|=\mathrm{O}\left(\exp \left(\exp \left(\frac{\pi \rho}{2 d} x\right)\right)\right) \quad(x \rightarrow \infty)
$$

holds uniformly with respect to $y \in(0, d)$, then we have

$$
\forall z \in \mathcal{Z}_{d}:|f(z)| \leq M .
$$

The following is the key lemma, which we derive from Corollary 7.4 above.

Lemma 7.5. For $d>0$ assume that a function $f: \overline{\mathcal{D}_{d}} \rightarrow \mathbf{C}$ is holomorphic on $\mathcal{D}_{d}$ and continuous on $\overline{\mathcal{D}_{d}}$, and that

$$
\forall z \in \overline{\mathcal{D}_{d}}:|f(z)| \leq M
$$

for a constant $M>0$. Also assume that

$$
\forall x \in \mathbf{R}:|f(x)| \leq M \exp (-\beta|x|)
$$

for a constant $\beta>0$. Then we have

$$
\forall x \in \mathbf{R}, \forall y \in \mathbf{R}(|y|<d):|f(x+\mathrm{i} y)| \leq M \exp \left(-\beta\left(1-\frac{|y|}{d}\right)|x|\right) .
$$

Proof. We assume $x \geq 0$ and $0 \leq y<d$ and define $F(z):=f(z) \omega(z)$ with

$$
\omega(z):=\exp \left(\beta\left(1+\mathrm{i} \frac{z}{2 d}\right) z\right) .
$$

Since

$$
|\omega(x+\mathrm{i} y)|=\left|\exp \left(\beta\left(1+\frac{-y+\mathrm{i} x}{2 d}\right)(x+\mathrm{i} y)\right)\right|=\exp \left(\beta\left(1-\frac{y}{d}\right) x\right),
$$

we have $|F(z)| \leq M$ for all $z \in \partial \mathcal{Z}_{d}$. In addition, for sufficiently large $x$, we have

$$
|F(x+\mathrm{i} y)| \leq M \exp (\beta x) \leq M \exp \left(\exp \left(\frac{\pi \rho}{2 d} x\right)\right)
$$

with $\rho>0$. Therefore, by Corollary [7.4, we obtain

$$
\forall z \in \mathcal{Z}_{d}:|F(z)| \leq M \text {. }
$$

Finally, we note that

$$
|f(z)|=|F(z)| /|\omega(z)| \leq M \exp \left(-\beta\left(1-\frac{y}{d}\right) x\right) .
$$

Thus we are done with the case where $x \geq 0$ and $0 \leq y<d$. Other cases, with $x \leq 0$ and/or $0 \geq y>-d$, can be treated in a similar manner. 
With the lemma above we can prove Theorem 7.2 as follows. By the assumptions, $f$ is holomorphic and bounded on $\overline{\mathcal{D}_{d-\varepsilon / 4}}$. It then follows from Lemma 7.5 that there exists a constant $M>0$ such that

$$
\forall z \in \overline{\mathcal{D}_{d-\varepsilon / 2}}:|f(z)| \leq M \exp \left(-\beta \frac{\varepsilon / 4}{d-\varepsilon / 4}|\operatorname{Re} z|\right) .
$$

This implies $f \in \mathbf{H}^{1}\left(\mathcal{D}_{d-\varepsilon / 2}\right)$ by Lemma 5.2

The rest of the proof is similar to that of Theorem 7.1. Just as for (7.4) we have

$$
\begin{aligned}
\sup _{-\infty<x<\infty}\left|f(x)-\sum_{k=-\infty}^{\infty} f(k h) S(k, h)(x)\right| & \leq C_{\varepsilon}^{\prime} \exp \left(-\frac{\pi(d-\varepsilon / 2)}{h}\right) \\
& =C_{\varepsilon}^{\prime} \exp \left(-\sqrt{\pi\left((d-\varepsilon / 2)^{2} / d\right) \beta N}\right) \\
& \leq C_{\varepsilon}^{\prime} \exp (-\sqrt{\pi(d-\varepsilon) \beta N})
\end{aligned}
$$

for the sampling error. For the truncation error we have

$$
\sup _{-\infty<x<\infty}\left|\sum_{|k|>N} f(k h) S(k, h)(x)\right| \leq \frac{2 \alpha}{\beta h} \exp (-\beta N h)=\frac{2 \alpha \sqrt{N}}{\sqrt{\pi \beta d}} \exp (-\sqrt{\pi d \beta N}),
$$

the same estimate as in (7.5). Hence follows (7.7). This completes the proof of Theorem 7.2 .

\section{REFERENCES}

[1] R. P. Boas, Entire Functions, Academic Press, New York, 1954. MR0068627(16:914f)

[2] J. McNamee, F. Stenger, and E. L. Whitney, Whittaker's cardinal function in retrospect, Math. Comp., 25 (1971), 141-154. MR0301428

[3] M. Mori and M. Sugihara, The double exponential transformations in numerical analysis, J. Comput. Appl. Math., 127 (2001), 287-296. MR1808579 (2001k:65041)

[4] M. Muhammad and M. Mori, Double exponential formulas for numerical indefinite integration, J. Comput. Appl. Math., 161 (2003), 431-448. MR2017024

[5] F. Stenger, Approximations via Whittaker's cardinal function, J. Approx. Theory, 17 (1976), 222-240. MR0481786

[6] F. Stenger, Numerical Methods Based on Sinc and Analytic Functions, Springer-Verlag, New York, 1993. MR1226236 (94k:65003)

[7] F. Stenger, Summary of Sinc numerical methods, J. Comp. Appl. Math., 121 (2000), 379-420. MR.1780056 (2001d:65018)

[8] M. Sugihara, Sinc approximation using double exponential transformations (in Japanese), Kokyuroku, Research Institute for Mathematical Sciences, Kyoto University, 990 (1997), 125-134. MR 1608743

[9] M. Sugihara, Optimality of the double exponential formula - functional analysis approach, Numer. Math., 75 (1997), 379-395. MR.1427714 (97i:41041)

[10] M. Sugihara, Near optimality of the Sinc approximation, Math. Comp., 72 (2003), 767-786. MR.1954967 (2004a:41026)

[11] M. Sugihara and T. Matsuo, Recent developments of the sinc numerical methods, J. Comput. Appl. Math., 164-165 (2004), 673-689. MR2056907

[12] H. Takahasi, M. Mori, Double exponential formulas for numerical integration, Publ. RIMS Kyoto Univ., 9 (1974), 721-741. MR0347061 (49:11781)

[13] K. Tanaka, M. Sugihara, and K. Murota, Numerical indefinite integration by double exponential Sinc method, Math. Comp., 74 (2005), 655-679. MR2114642(2006a:65035)

[14] K. Tanaka, M. Sugihara, and K. Murota, Function classes for successful DE-Sinc approximations, Technical report METR 2007-08, University of Tokyo, February 2007. 
Department of Mathematical Informatics, Graduate School of Information Science and Technology, University of Tokyo, 7-3-1, Hongo, Bunkyo-ku, Tokyo, 113-8656, Japan

Department of Mathematical Informatics, Graduate School of Information Science and Technology, University of Tokyo, 7-3-1, Hongo, Bunkyo-Ku, Tokyo, 113-8656, Japan

E-mail address: m_sugihara@mist.i.u-tokyo.ac.jp

Department of Mathematical Informatics, Graduate School of Information Science and Technology, University of Tokyo, 7-3-1, Hongo, Bunkyo-Ku, Tokyo, 113-8656, Japan

E-mail address: murota@mist.i.u-tokyo.ac.jp 